\title{
Avaliação e intervenção no Transtorno da Compulsão Alimentar (TCA): uma revisão sistemática
}

\author{
Giovanna Nunes Cauduro ${ }^{1}$ \\ Gabriel Machado Paz $^{2}$ \\ Janaína Thaís Barbosa Pacheco ${ }^{2}$ \\ ${ }^{1}$ Universidade Federal do Rio Grande do Sul, RS, Brasil \\ ${ }^{2}$ Universidade Federal de Ciências da Saúde de Porto Alegre, RS, Brasil
}

\begin{abstract}
Resumo
O transtorno da compulsão alimentar (TCA) é descrito no DSM-V como episódios recorrentes de ingestão de grandes quantidades de alimentos, seguidos por desconforto físico, sofrimento emocional e sensação de perda de controle. Este artigo objetivou identificar as características metodológicas dos estudos, descrever as técnicas de intervenção e os instrumentos de avaliação utilizados no tratamento do TCA. Foi conduzida uma revisão sistemática da literatura de estudos empíricos nacionais e internacionais, utilizando as bases de dados Lilacs e Scielo, PubMed, PsycInfo e Science Direct para consulta. Foram encontrados 176 artigos, dos quais 33 preencheram os critérios de inclusão. A Terapia Cognitiva Comportamental apareceu com maior frequência. Os principais instrumentos utilizados para avaliação deste quadro foram o Eating Disorder Examination (EDE) e o Escala de Depressão de Beck (BDI). A partir dos resultados, foi possível identificar uma tendência de mudança quanto à abordagem utilizada, concomitante à necessidade de avaliação de comorbidades do quadro.
\end{abstract}

Palavras-chave: Intervenção psicológica; Avaliação; Compulsão alimentar; Transtornos alimentares.

\section{Assessment and intervention in Binge Eating Disorder (BED): a systematic review}

\section{Abstract}

The binge eating disorder is described in DSM-V as recurrent ingestion episodes of big amounts of food, followed by physical discomfort, emotional suffer and out of control feeling. The article focused on identify studies' methodologic characteristics, describe interventions technics and evaluate tools on the TCA treatment. A systematic literature review with national and international empiric studies was conducted, consulting Lilacs, Scielo, Pubmed, Psycinfo and Science Direct as data base. 176 articles were found and 33 of them filled the inclusion criteria. The cognitive-behavior therapy had the biggest frequency. The main evaluation tools used were Eating Disorder Examination (EDE) and Beck Depression Inventory Scale. From the results is possible to identify a tendency of changes about the utilized approach, concomitant to the necessary evaluation of disorder comorbidity.

Keywords: Psychological intervention; Assessment; Binge eating; Eating disorders.

\section{Evaluación e intervención en el Trastorno por Atracón (TA): una revisión sistemática}

\section{Resumen}

El trastorno por atracón se describe en el DSM-V como episodios recurrentes de ingestión de grandes cantidades de alimentos, seguidos de incomodidad física, sufrimiento emocional y sensación de descontrol. El artículo se enfocó en identificar las características metodológicas de los estudios, describir las técnicas de intervención y evaluar las herramientas en el tratamiento de TCA. Se realizó una revisión sistemática de la literatura con estudios empíricos nacionales e internacionales, consultando a Lilacs, Scielo, Pubmed, Psycinfo y Science Direct como base de datos. Se encontraron 176 artículos y 33 de ellos cumplieron los criterios de inclusión. La terapia de comportamiento cognitivo tuvo la mayor frecuencia. Las principales herramientas de evaluación utilizadas fueron el Examen del trastorno alimentario (EDE) y la Escala de inventario de depresión de Beck. De los resultados es posible identificar una tendencia de los cambios sobre el enfoque utilizado, concomitante a la necesaria evaluación de la comorbilidad del trastorno.

Palabras clave: Intervención psicológica; evaluación; atracones; trastornos de la alimentación. 


\section{Introdução}

Os Transtornos Alimentares são um grupo de transtornos mentais severos, incapacitantes e frequentemente crônicos (Klump, Bulik, Kaye, Treasure, \& Tyson, 2009). Esses transtornos são caracterizados pela perturbação persistente no comportamento alimentar, ocasionando alterações no consumo e na absorção dos alimentos (American Psychiatric Association, 2013). Atualmente, são considerados Transtornos Alimentares: Pica; Ruminação; Evitação/Restrição da ingestão alimentar, Anorexia Nervosa (AN), Bulimia Nervosa (BN) e Transtorno da Compulsão Alimentar (TCA; APA, 2013).

O Transtorno da Compulsão Alimentar Periódica (TCAP) foi descrito inicialmente por Stunkard (1959) como a ingestão de grandes quantidades de alimentos em um curto período de tempo, seguido, geralmente, de alto desconforto e autocondenação. Em 1994, o TCAP aparece descrito na quarta edição do Manual Diagnóstico e Estatístico de Transtornos Mentais (DSM-IV-TR, APA, 2002) como um dos seis transtornos alimentares sem outra especificação. Desde então, o TCAP vem passando por alterações quanto aos critérios diagnósticos nas publicações do DSM.

Em 2013, na publicação do DSM-V (APA, 2013), o TCAP foi finalmente descrito como um transtorno alimentar específico com categoria diagnóstica própria. Entre as versões IV-TR e V do DSM, os critérios diagnósticos anteriormente descritos para pesquisa foram modificados. No DSM-IV-TR, o paciente recebia o diagnóstico caso apresentasse episódios de compulsão alimentar (ECA) duas vezes na semana, por, no mínimo, seis meses.No DSM-V, é diagnosticado o paciente que apresentar ao menos um ECA por semana, durante no mínimo três meses. Além da alteração destes critérios, o transtorno passou a ser denominado Transtorno da Compulsão Alimentar (TCA) (APA, 2013).

Estudos recentes vem investigando fatores antecedentes aos episódios de compulsão alimentar na tentativa de identificar os gatilhos que levam os pacientes a ingerirem elevadas quantidades de comida em um período de tempo limitado. Em um estudo realizado por Boggianoet al. (2016), aspectos emocionais, principalmente os de valência negativa, podem estar relacionados a episódios de comer compulsivo. Os autores apontam que o repertório individual do paciente para lidar com contextos potencialmente estressantes pode aumentar as chances da ingestão calórica excessiva quando comparada a outros indivíduos em um contexto semelhante. Isso porque o tipo de alimento ingerido durante esses períodos tende a ser altamente palatável, atuando como reforço negativo, quando diminui a sensação de emoções negativas (tristeza, estresse, etc.), por exemplo.

Transtornos alimentares tendem a afetar uma população mais jovem (Treasure, Claudino, \& Zucker, 2010) sendo que, entre crianças estado-unidenses, com idade entre 9 e 14 anos, a prevalência de comportamentos alimentares disfuncionais é de $7,1 \%$ em meninos e $13,4 \%$ em meninas (Neumark-Sztainer \& Hannan, 2000). Em adultos, a prevalência dos transtornos alimentares em adultos é de $0,6 \%$ para anorexia nervosa, $1 \%$ para bulimia nervosa e 3\% para transtorno da compulsão alimentar. Além disto, cabe ressaltar que essa diferença de prevalência é expressiva entre os gêneros, sendo as mulheres mais afetadas por estes transtornos (Jacobi, Wittchen, \& Holting, 2004; Hudson, Hiripi, Pope, \& Kessler, 2007).

Devido à alta prevalência do transtorno, diferentes estratégias terapêuticas vem sendo utilizadas no tratamento do TCA. Entre elas destacam-se as intervenções baseadas na abordagem CognitivoComportamental, cuja modalidade terapêutica e técnicas de modificação vêm sendo amplamente utilizadas, tanto para o tratamento do TCA, quanto de suas comorbidades (Yager \& Powers, 2009). No entanto, novas abordagens e técnicas vêm sendo implementadas e avaliadas quanto sua eficácia na redução de ECAs e no aumento da taxa de remissão do transtorno (Chen et al., 2017). Modalidades interventivas usadas para tratamento de outros transtornos da adição também passaram a ser consideradas devido à semelhança que apresentam (Schulte \& Gearhardt, 2016).

Considerando as mudanças nos critérios diagnósticos do TCA e os estudos sobre intervenções terapêuticas para esse transtorno, esse artigo objetivou descrever os instrumentos de avaliação psicológica diagnóstica utilizados nos estudos sobre TCA e as técnicas empregadas nas intervenções psicológicas realizadas com portadores de Transtorno da Compulsão Alimentar.Os objetivos específicos incluíram: 1) identificar os contextos mais comuns nos quais estas intervenções são utilizadas; 2) descrever o perfil dos pacientes participantes das intervenções e 3) revisar aspectos metodológicos da produção científica sobre o assunto. Para isso, conduziu-se uma revisão sistemática da literatura de estudos empíricos nacionais e internacionais sobre Transtorno da Compulsão Alimentar.

\section{Método}

Esta pesquisa baseou-se no método de revisão sistemática da literatura proposto por Wright, Brand, Dunn e Spindler (2007). As bases de dados 
internacionais consultadas foram PubMed, PsycInfo e Science Direct utilizando-se os descritores "binge eating disorder", "intervention OR therapy" e "assessment". As bases Lilacs e Scielo foram consultadas para identificar estudos nacionais e a pesquisa utilizou os descritores "transtorno da compulsão alimentar", "intervenção OU terapia" e "avaliação". Em ambos os casos os descritores poderiam estar presentes no título ou resumo. Foram utilizados limitadores de idiomas (estudos em português, espanhol e inglês) e pesquisa com humanos. Não foi utilizada restrição de período na busca.A consulta foi realizada em novembro de 2016. No total foram identificados 176 artigos.

Os 176 resumos foram lidos por dois pesquisadores independentes e foram aplicados os seguintes critérios de exclusão: a) estudos teóricos ou de revisão da literatura; b) estudos sem intervenção psicológica; c) estudos que apresentassem somente episódios isolados de compulsão alimentar ou episódios dentro do transtorno bulímico; d) estudos cujos participantes não foram diagnosticados com TCA; e) estudos sem utilização de instrumentos de avaliação de TCA. Com aplicação dos critérios de exclusão, 133 estudos foram retirados da revisão. Dessa forma, 43 artigos foram considerados para análise dos dados por atenderem os seguintes critérios de inclusão: a) presença de intervenção psicológica ou psicoterápica; b) diagnóstico formal de TCA e c) utilização de instrumentos de avaliação. Destes, 10 foram excluídos após a leitura na íntegra por não responderem aos critérios de inclusão mencionados acima. Restaram, então, 33 artigos considerados para a extração dos dados.

A etapa de extração dos dados foi realizada por dois pesquisadores independentes a partir de um protocolo pré-estabelecido de extração. Desse protocolo foram extraídos os seguintes dados de todos os artigos incluídos na revisão: autores; título do artigo; ano de publicação; país do estudo; objetivo; público alvo; tamanho da amostra; delineamento; contexto no qual a intervenção foi aplicada; tipo de intervenção (individual ou em grupo); abordagem teórica; número de sessões; principais estratégias terapêuticas; instrumentos utilizados; e principais resultados. Ao término da extração, a tabela foi revisada por um terceiro pesquisador para evitar possíveis equívocos. Para ilustrar as etapas do presente estudo, foi elaborado um fluxograma contendo todas as etapas de inclusão e exclusão de artigos (Figura 1).

\section{Resultados}

Os resultados encontrados a partir da extração dos 33 artigos serão reportados de acordo com os

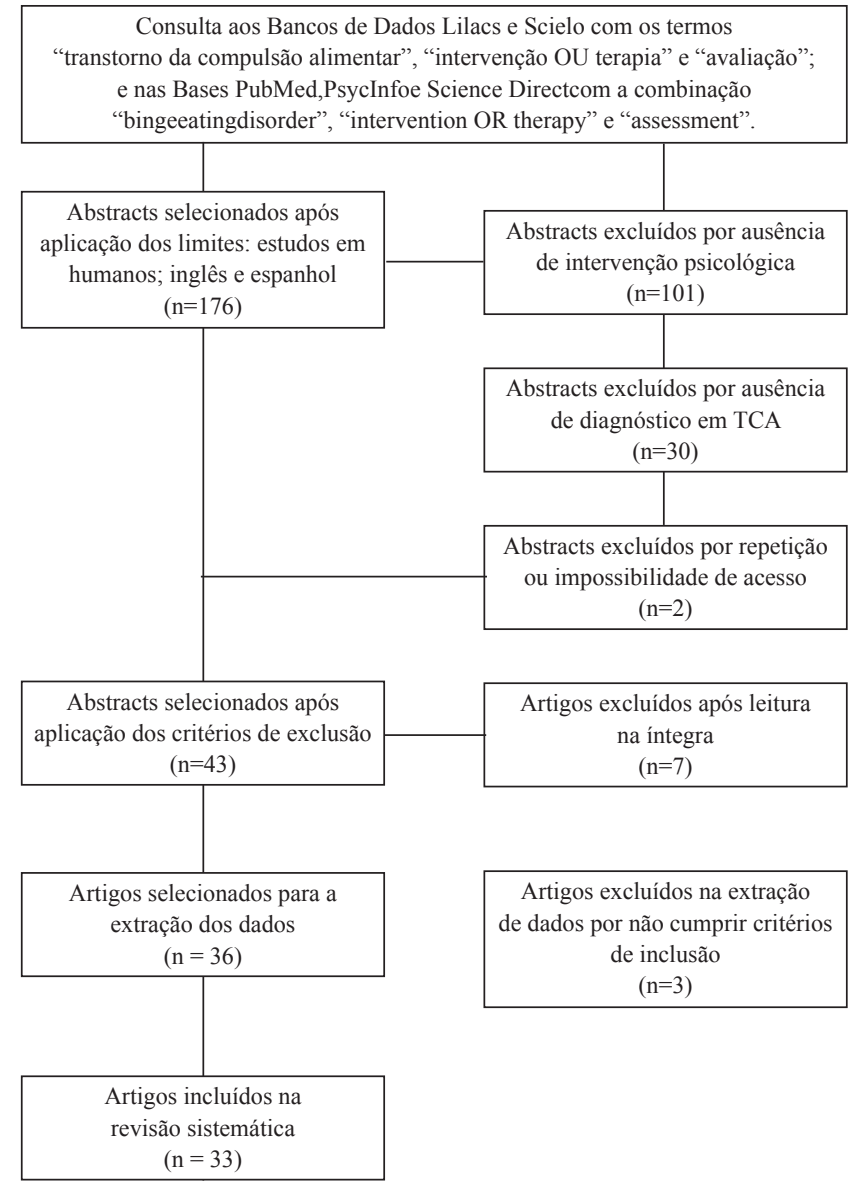

Figura 1. Fluxograma

objetivos do estudo. Serão dispostos de maneira a: 1) identificar características metodológicas dos estudos; 2) descrever as abordagens e estratégias de intervenção encontrados; e 3) descrever os instrumentos utilizados para o diagnóstico e a avaliação do Transtorno da Compulsão Alimentar.

\section{Características metodológicas}

\section{Delineamentos}

A Tabela 1 reúne as características metodológicas dos 33 artigos utilizados na extração do estudo. Nessa tabela, são descritos os nomes dos autores do estudo; ano e país de publicação; tamanho da amostra (grupo de participantes do estudo e grupo controle, quando aplicável); delineamento do estudo e contexto no qual a intervenção foi realizada. A numeração dos artigos na Tabela 1 servirá de referência aos estudos para o resto da leitura do artigo.

Quanto à origem dos estudos incluídos na presente revisão, $23(69,7 \%$; 1, 3, 6, 8, 9, 10, 11, 14, 16, 17, 19, $20,21,23,24,25,26,27,29,30,31,32,33)$ foram realizados nos Estados Unidos, seguidos por quatro 
TABELA 1

Características metodológicas dos estudos incluídos na revisão

\begin{tabular}{|c|c|c|c|c|c|c|}
\hline & \multirow{2}{*}{ Autores } & \multirow{2}{*}{ Ano } & \multirow{2}{*}{$\begin{array}{c}\text { Pais do } \\
\text { estudo }\end{array}$} & \multicolumn{2}{|c|}{ Tamanho da amostra } & \multirow{2}{*}{ Delineamento } \\
\hline & & & & Intervenção & Controle & \\
\hline 1 & Manasse et al. & 2016 & EUA & 19 & 17 & Estudo piloto de amostragem por conveniência \\
\hline 2 & Wagner et al. & 2016 & Alemanha & 69 & 70 & Ensaio Clinico Randomizado \\
\hline 3 & Hill et al. & 2015 & EUA & 2 & & Não especificado \\
\hline 4 & Svaldi et al. & 2014 & Alemanha & 19 & 20 & Não especificado \\
\hline 5 & Vancampfort et al. & 2014 & Bélgica & 34 & 31 & Não especificado \\
\hline 6 & Grilo et al. & 2014 & EUA & 52 & 52 & Ensaio clínico randomizado controlado com placebo \\
\hline 7 & Brauhardt et al. & 2014 & Alemanha & 89 & & Ensaio Clinico Randomizado \\
\hline 8 & Peterson et al. & 2013 & EUA & 190 & 69 & Não especificado \\
\hline 9 & Robinson et al. & 2013 & EUA & 16 & 12 & Não especificado \\
\hline 10 & Grilo et al. & 2013 & EUA & 40 & 39 & Ensaio Clínico Randomizado \\
\hline 11 & Grilo et al. & 2013 & EUA & 24 & 24 & Ensaio clínico randomizado controlado com placebo \\
\hline 12 & Munsch, Meyer, Biedert & 2012 & Suiça & 44 & 36 & Ensaio Randomizado Comparativo \\
\hline 13 & Flückiger et al. & 2011 & Suiça & 28 & 23 & Ensaio Clinico Randomizado \\
\hline 14 & Masheb, Grilo, Rolls & 2011 & EUA & 25 & 25 & Ensaio Clínico Randomizado \\
\hline 15 & Carrard et al & 2011 & Suiça & 37 & 37 & Ensaio Clínico Randomizado \\
\hline 16 & Safer, Joyce & 2011 & EUA & 50 & 51 & Ensaio Clínico Randomizado \\
\hline 17 & Grilo et al. & 2011 & EUA & 125 & & Ensaio Clínico Randomizado \\
\hline 18 & Ricca et al. & 2010 & Itália & 72 & 72 & Ensaio Clínico Randomizado \\
\hline 19 & Safer, Jo & 2010 & EUA & 50 & 51 & Ensaio Clínico Randomizado \\
\hline 20 & Masheb, Grilo & 2008 & EUA & 37 & 38 & Ensaio Clínico Randomizado \\
\hline 21 & Safer, Couturier \& Lock & 2007 & EUA & 1 & & Estudo de caso \\
\hline 22 & Ljotsson & 2007 & Suécia & 36 & 36 & Ensaio Clínico Randomizado \\
\hline 23 & Grilo et al. & 2007 & EUA & 25 & 25 & Ensaio Clínico Randomizado \\
\hline 24 & Grilo et al. & 2005 & EUA & 25 & 25 & Ensaio randomizado duplo cego controlado com placebo \\
\hline 25 & Grilo et al. & 2005 & EUA & 74 & 15 & Ensaio Clínico Randomizado \\
\hline 26 & Grilo et al. & 2005 & EUA & 54 & 54 & Ensaio randomizado duplo cego controlado com placebo \\
\hline 27 & deZwaan & 2005 & EUA & 71 & 83 & Ensaio Clínico Randomizado \\
\hline 28 & Hilbert & 2004 & Alemanha & 14 & 14 & Não especificado \\
\hline 29 & Latner & 2002 & EUA & 30 & & Não especificado \\
\hline 30 & Telch, Linehan & 2000 & EUA & 11 & & Ensaio clínico não controlado \\
\hline 31 & Allen & 1999 & EUA & 11 & 9 & Não especificado \\
\hline 32 & Gladis & 1998 & EUA & 118 & & Ensaio Clínico Randomizado \\
\hline 33 & Craighead, Wilcoxon & 1995 & EUA & 3 & & Estudo de Caso \\
\hline
\end{tabular}

$(12,1 \% ; 2,4,7,28)$ realizados na Alemanha, e três $(9 \% ; 12,13,15)$ na Suíça. Pesquisas ocorridas na Itália, Suécia e Bélgica apareceram somente uma vez cada $(18,22,5$, respectivamente). No que tange o delineamento das pesquisas, destaca-se que 16 $(48,4 \% ; 2,7,10,13,14,15,16,17,18,19,20,22$, 23, 25, 27, 32) realizaram Ensaios Clínicos Randomizados (ECR); quatro $(12,1 \% ; 6,11,24,26)$ realizaram ECR controlado com placebo e dois $(6 \% ; 21,33)$ optaram por descrever o fenômeno através de Estudos de Caso. Ademais, apenas um optou por realizar um estudo piloto com amostragem por conveniência
$(3 \%, 1)$; outro optou por realizar um Ensaio Randomizado Comparativo $(3 \% ; 12)$ e outro por realizar um Ensaio Clínico Não-Controlado $(3 \% ; 30)$. Outros oito $(24,2 \% ; 3,4,5,8,9,28,29,31)$ não especificam em seus métodos o delineamento escolhido. Na Figura 2, estão dispostos os tipos de delineamento de estudos publicados ao longo do período pesquisado nesta revisão. Percebe-se que a escolha metodológica dos estudos independeu do ano de publicação, uma vez que estudos utilizando ECR, por exemplo, incluídos nesta revisão a estão datados a partir de 1998. 


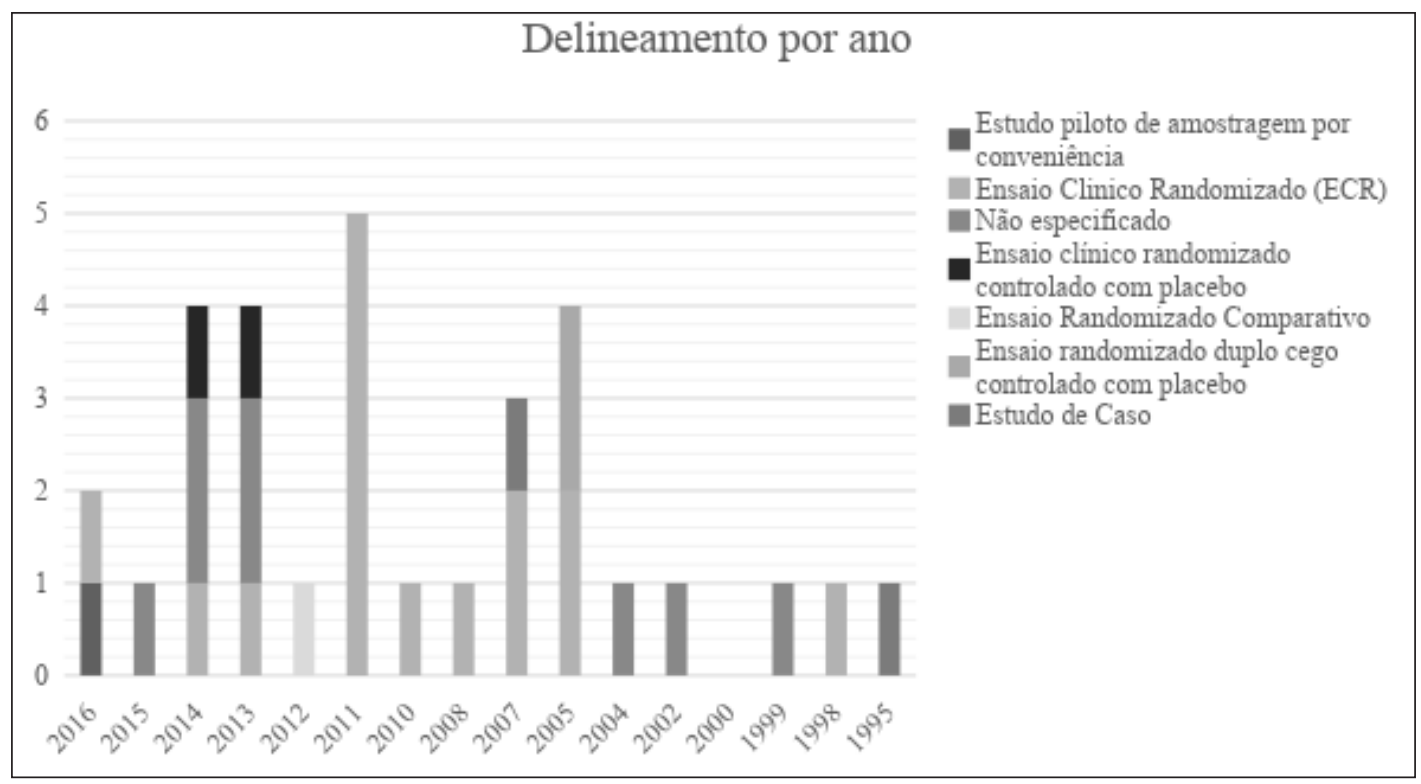

Figura 2. Delineamento de acordo com o ano de publicação dos estudos

\section{Caracterização da amostra}

Informações relacionadas às características da amostra utilizada nos estudos foram extraídas para fins de caracterização da amostra. Para isso, dados como sexo, faixa etária, diagnóstico, critérios para inclusão e exclusão para participação nas pesquisas foram analisados. Dos 33 artigos incluídos na presente revisão, nove $(30,3 \%)$ foram realizados somente com participantes do sexo feminino, enquanto $22(66,6 \%)$ envolveram indivíduos tanto do sexo feminino quanto do sexo masculino. Destes, apenas um estudo foi realizado com adolescentes, os demais foram direcionados a investigar indivíduos adultos.

Os critérios de inclusão variaram entre pesquisas, sendo a principal variação relacionada ao diagnóstico necessário para participação nos estudos. Ter somente diagnóstico de TCA foi fator necessário em 15 $(45,4 \%)$ dos estudos incluídos; ser diagnosticado com TCA e apresentar critérios para a obesidade foi fator de inclusão para $16(48,4 \%)$ estudos; enquanto dois $(6 \%)$ estudos requereram que os participantes tivessem diagnóstico de TCA associado ao sobrepeso. A presença de diagnóstico de outros transtornos psiquiátricos ou condições físicas clínicas severas foi o critério de exclusão mais comumente aplicado aos estudos incluídos nesta revisão.

Dos estudos participantes desta pesquisa, 24 utilizaram grupos controle, representando $72,7 \%$ da amostra $(1,2,3,4,5,6,7,8,9,10,11,12,13,14,15$, $16,17,18,19,20,21,22,23,24,25,26,27,28,29$, $30,31)$; outras quatro pesquisas $(15,1 \%)$ incluíam dois ou mais grupos de intervenção, além do grupo controle $(7,17,27,29)$. Dos artigos incluídos, três $(9 \% ; 3,21$, 32) descreveram estudos de caso. Apenas um artigo (30) apresentou um estudo de comparação entre dois grupos clínicos distintos - Bulimia Nervosa (BN) e Transtorno da Compulsão Alimentar (TCA) - buscando verificar a efetividade de tratamentos específicos em ambas as condições. O tamanho amostral variou de 19 a 190 sujeitos nos grupos de intervenção, e 9 a 83 participantes nos grupos controle. Nos estudos com mais de dois grupos interventivos houve uma variação de 11 a 125 sujeitos. Nos estudos de caso ocorreu uma diferenciação de 1 a 3 participantes.

\section{Objetivos dos estudos analisados}

A fim de organizar a análise dos dados referentes aos objetivos dos artigos incluídos nesta revisão, optou-se pela inclusão desses em clusters criados a partir da semelhança entre os objetivos de pesquisa. Dois estudos (17 e 19) apresentaram mais de um objetivo. Os objetivos foram agrupados em seis categorias, sendo: 1) verificar a eficácia do tratamento; 2) associar variáveis relacionadas ao fenômeno; 3) comparar intervenções; 4) predizer o resultado do tratamento; 5) verificar a eficácia das intervenções medicamentosas e psicoterápicas; e 6) outros. Desta maneira, verificou-se que $48 \%$ dos estudos $(2,5,6,10,11,15,21,22,23,25,27,29,30$, $32,33)$ foram incluídos no cluster número $1 ; 18 \%(7,18$, $19,20,28,31)$ incluídos no cluster número $3 ; 18 \%(1,8$, $12,16,17,18)$ relacionavam-se ao tema geral do cluster número 4. Os clusters 2, 5 e 6 representaram, cada, 9\% $(3,4,13,14,14,26)$ da amostra. 


\section{Características das Intervenções}

\section{Tipo de intervenção}

A intervenção individual foi a mais frequente nos estudos analisados, tendo sido utilizada em 29 estudos, ou seja, 45,4\% da amostra $(2,3,4,7,14,15,20,21$, $22,23,24,25,26,28$ e 29). Subsequente a isso, as intervenções realizadas em grupo foram a opção de $33,3 \%$ dos artigos incluídos $(1,5,8,9,12,17,27$, $30,31,32$ e 33). Pesquisas cujas intervenções foram de cunho misto, ou seja, com etapas individuais e etapas grupais, representaram apenas $12,1 \%$ da amostra e são descritas nos artigos de número 13,16, 18 e 19. Três estudos $(6,10$ e 11) não especificam se as intervenções foram individuais, grupais ou mistas, representando $9 \%$ dos artigos utilizados.

\section{Estratégias terapêuticas}

No que diz respeito às estratégias terapêuticas selecionadas para o tratamento do Transtorno da Compulsão Alimentar nos artigos revisados, é importante ressaltar que todos associaram uma ou mais estratégias, de acordo com os objetivos de cada estudo. A abordagem psicoterápica mais utilizada foi a Terapia Cognitiva Comportamental (TCC), aparecendo em 23 estudos $(69,6 \% ; 2,4,5,6,7,8,11,12,13,14$, $15,17,18,20,22,23,24,25,27,28,29,31,32)$, dentre os quais $12(52,1 \% ; 2,4,7,14,15,20,22,23$, $24,25,28,29)$ consistiam em estudos descrevendo a TCC em intervenções individuais, sete $(30,4 \% ; 5,8$, $12,17,27,31,32)$ detalhando intervenções grupais e dois $(8,6 \% ; 13,18)$ apresentando intervenções mistas, com etapas individuais e grupais. Dentre as técnicas mais utilizadas, em primeiro lugar aparece a Psicoeducação, presente em 33,3\% dos estudos; seguida pelo Automonitoramento, utilizada em 30,3\% das pesquisas. Na Figura 3 estão dispostas a frequência de todas as estratégias conforme descritas nos artigos analisados.

Dos 23 artigos nos quais foi referido o uso da Terapia Cognitivo-Comportamental enquanto abordagem terapêutica, 14 (82,3\%) optaram por empregar técnicas comuns da abordagem como principal ferramenta de seus estudos. Dentre esses que se propuseram a explicar de forma detalhada as estratégias, notou-se que as intervenções foram estruturadas, geralmente compostas por material informativo para fins de psicoeducação; tarefas de escrita visando o auto monitoramento; e acompanhamento com terapeuta para manutenção dos ganhos terapêuticos. Recursos adicionais como materiais audiovisuais foram utilizados, mas apareceram em menor frequência. Apesar das técnicas serem as mesmas, elas diferiram entre si nos meios através dos quais eram realizadas (e.g., presencial, on-line, através de protocolos impressos para preenchimento em casa). No entanto, o caráter focal visando promover o desenvolvimento de autonomia do paciente foi comum a todas as pesquisas que descreveram o uso de tais estratégias.

O número de encontros, tempo das sessões, materiais utilizados, objetivo de cada encontro e demais características dos tratamentos apareceram de modo semelhante nos estudos que optaram pela TCC. Os números de sessões variaram de 1 a 38 encontros.

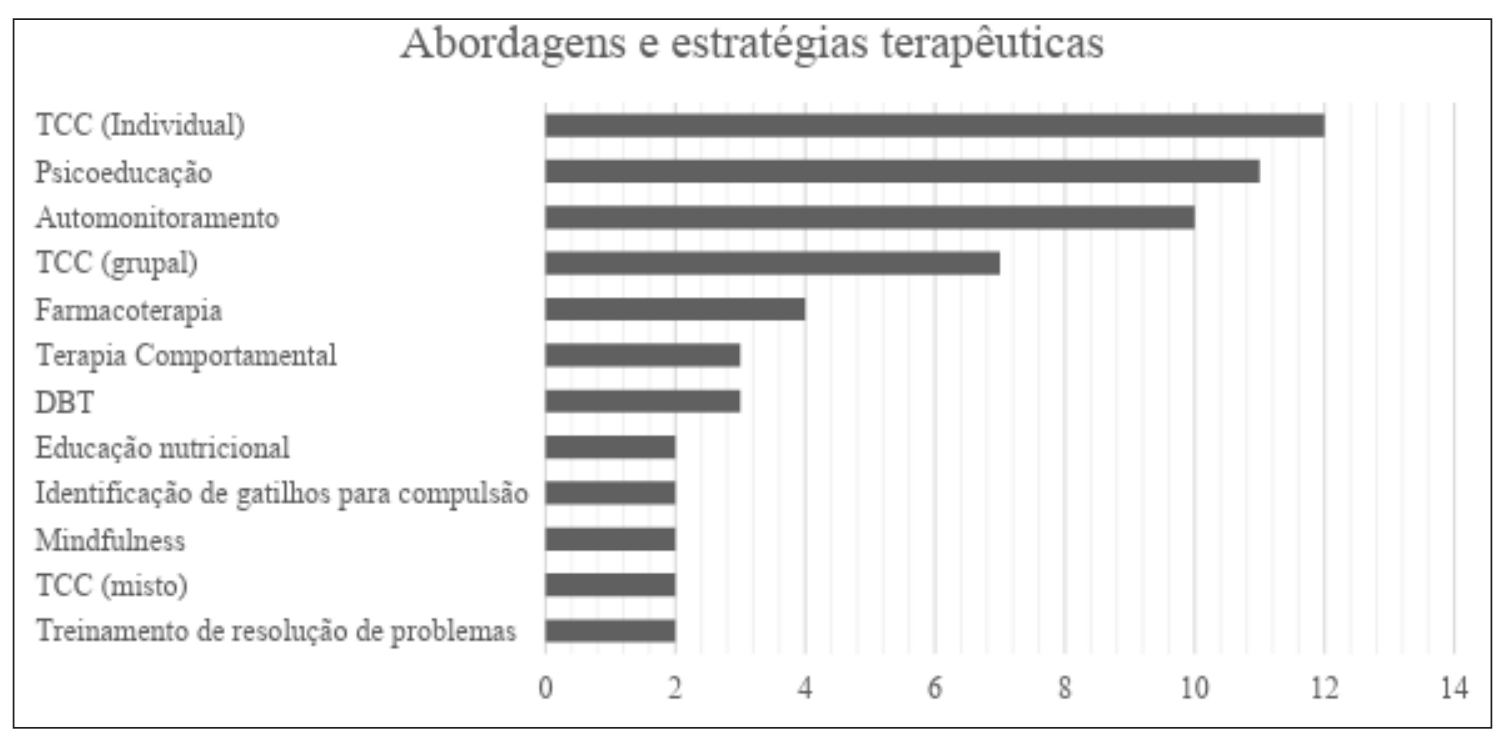

Figura 3. Principais estratégias terapêuticas utilizadas nas intervenções 
Sendo que, dentre os materiais utilizados, o livro de Fairburn (1995), "Overcoming Binge Eating", um manual de autoajuda para o tratamento de TCA apareceu frequentemente como ferramenta de tratamento. No entanto, é clara a diferença entre estudos que se dá pelos fatores idiossincráticos das amostras utilizadas e pelos objetivos de cada estudo.

\section{Principais resultados encontrados nos estudos revisados}

Para fins de descrição dos principais resultados, levou-se em consideração os clusters de objetivos descritos anteriormente. Por essa razão, serão descritos aqui os resultados dos artigos incluídos nas categorias: 1) verificação de eficácia de tratamento, na qual $48 \%$ dos estudos foram incluídos; 3) comparação de intervenções, referente ao objetivo de $18 \%$ dos artigos; e 4) predição dos resultados do tratamento, que englobou, também, 18\% dos objetivos dos estudos. Os resultados dos demais artigos não serão incluídos nessa análise uma vez que apareceram em menos de $10 \%$ da amostra da presente pesquisa.

Dos 15 artigos que se propuseram a verificar a eficácia de diferentes estratégias terapêuticas no tratamento do transtorno da compulsão alimentar, apenas seis $(2,5,21,23,25,27)$ encontraram diferenças significativas na diminuição dos episódios de CA após as intervenções propostas. O estudo de Grilo e Masheb (2005) apresentou diferenças significativas $(p=0.01)$ nas taxas de remissão entre as condições de tratamento: taxa de remissão de $13,3 \%$ nos grupos controle; $18,4 \%$ nos grupos que receberam tratamento do tipo behavioralweightloss; e 46\% nos grupos que receberam intervenção baseada na abordagem cognitivo comportamental.

Safer, Lock e Couturier (2007) descreveram um estudo de caso no qual a Terapia Comportamental Dialética foi adaptada e utilizada com uma adolescente de 16 anos do sexo feminino. Os autores descreveram detalhadamente o conteúdo das sessões, agrupandoas por assuntos em comum. Antes da intervenção a adolescente havia apresentado 22 episódios de compulsão nos 28 dias antecedentes. Após a intervenção, esse número reduziu a quatro episódios no mês subsequente e um episódio nos três meses seguintes indicando uma redução significativa dos episódios de compulsão alimentar em si.

Dentre os estudos que compararam métodos de intervenção, apenas um (18) obteve diferenças significativas entre modelos de tratamento. Ricca et al. (2010) compararam as modalidades de grupo e individual de intervenção de Terapia CognitivoComportamental em um estudo randomizado com avaliação de follow-up após 3 anos do tratamento. Ambos os modelos de intervenção indicaram redução significativa no número de episódios de compulsão alimentar e de peso corporal ao final dos 24 meses (T1). No entanto, na avaliação realizada ao final dos três anos de estudo (T2), a redução significativa de ECA, dos escores no Inventário de Depressão de Beck (BDI) e no Eating Disorder Examination Questionnaire (EDE-Q) foi identificada nos pacientes que foram incluídos na intervenção grupal.

Seis artigos tiveram como objetivo investigar os preditores de eficácia terapêutica para TCA. Desses, quatro $(1,12,16$ e 18) apresentaram resultados significativos para os preditores avaliados. Manasse et al (2016) encontrou que indivíduos com maior tendência para comer precipitadamente quando emocionalmente angustiados tiveram menores reduções nos ECA durante e após o tratamento, sugerindo baixa resposta ao tratamento. Tanto no estudo conduzido por Safer e Joyce (2011), quanto no de Munsch, Meyer e Biedert (2012), ficou evidente a relação entre indivíduos que apresentaram preditor de rápida resposta ao tratamento (rapid response) e a maior eficácia das intervenções. Ricca et al (2010) encontraram uma associação significativa entre TCA, sobrepeso na infância e resistência ao tratamento na idade adulta.

\section{Características dos Instrumentos de Avaliação Psicológica}

Foram relatados 55 diferentes instrumentos de avaliação psicológica diagnóstica utilizados nos 33 artigos incluídos. Apenas um estudo (22) utilizou apenas um instrumento para avaliar os participantes, os demais utilizaram duas ou mais ferramentas de avaliação. Dos 55 instrumentos encontrados, 41 $(81,8 \%)$ apareceram menos de duas vezes cada, e, portanto, não serão citados aqui.

Dentre os 55 instrumentos citados nos 33 artigos revisados, o EDE (Fairburn \& Cooper, 1987) foi o mais frequentemente utilizado para avaliar comportamentos alimentares relacionados ao TCA, representando $28 \%$ dos instrumentos descritos. O segundo instrumento mais utilizado nos estudos incluídos foi o BDI, ferramenta largamente usada na avaliação de sintomas depressivos, e representou $23 \%$ dos instrumentos citados. A Structured Clinical Interview for the DSM (SCID) apareceu em terceiro lugar entre os instrumentos escolhidos para avaliação de casos de TCA, representando $12 \%$ das ferramentas. Em quarto lugar, referindo-se a $8 \%$ dos instrumentos descritos, está a RosenbergSelf-EsteemScale(RSE; Rosenberg, 1979).

$\mathrm{O}$ Eating Disorder Examination (EDE) consiste em uma entrevista semiestruturada, elaborada e descrita 
inicialmente em um estudo de Cooper e Fairburn (1987). Essa ferramenta foi desenvolvida visando preencher uma lacuna deixada por outros instrumentos de autorrelato, uma vez que transtornos alimentares apresentam nuances relacionadas às crenças pessoais de peso e imagem corporal, e a técnica de entrevista tende a ser mais útil na compreensão adequada destas nuances. O objetivo inicial dessa técnica era diagnosticar quadros de Bulimia Nervosa e Anorexia Nervosa, mas tem sido amplamente utilizada para diagnosticar casos de TCA (Cooper \& Fairburn, 1987; Fairburn \& Beglin, 1993).

O Beck Depression Inventory (BDI) é um inventário desenvolvido por Beck, Ward, Mendelson, Mock e Erbaugh (1961) visando a mensuração de manifestações comportamentais da depressão. Esse inventário é composto por 21 itens de múltipla escolha desenvolvidos a partir de material clínico, frente à lacuna deixada por instrumentos semelhantes na época (Beck et al., 1961). Devido à qualidade de suas propriedades psicométricas este é um instrumento que vem sendo traduzido, adaptado e validado para diferentes culturas (Abdel-Khalek, 1998; Kapci, Uslu, Turkcapar \& Karaoglan, 2008; Gorestein \& Andrade, 1998).

First (2015) descreve a Structured Clinical Interview for the DSM (SCID) como uma entrevista clínica cuja estrutura é pensada a fim de facilitar o diagnóstico de doenças mentais a partir dos critérios do Manual Diagnóstico e Estatístico dos Transtornos Mentais (DSM). Essa entrevista varia conforme a edição do DSM, sendo adaptada a cada edição, acompanhando os critérios incluídos e excluídos (First, 2015; Spitzer, Williams, Gibbon \& First, 1992; Williams et al., 1992). A SCID pode ser customizada para cada uso, visando atender as necessidades específicas de investigação. Isso porque sua configuração permite utilizar as perguntas básicas para triagem de transtornos mentais em pacientes psiquiátricos não identificados, ou especificar o tipo e a gravidade do transtorno já identificado (First, 2015).

Composta por 10 itens dicotômicos, a Rosenberg Self-Esteem Scale (RSE) foi proposta inicialmente por Morris Rosenberg, em 1965 (Sinclair et al., 2010) e se propunha a mensurar autoestima em estudantes do ensino médio. No entanto, esse instrumento vem sendo utilizado amplamente em diversas faixas etárias, principalmente adultos (Ciarrochi \& Bilich, 2007). Os 10 itens são igualmente divididos em duas principais facetas do construto "autoestima": self-liking (SL) e self-competence (SC), nos quais SL se dá como um valor intrínseco, enquanto SC é apresentado como um valor instrumental (Tafarodi \& Milne, 2002).

\section{Discussão}

O presente estudo teve como finalidade descrever instrumentos de avaliação psicológica, estratégias psicoterapêuticas e as características metodológicas em pesquisas que investigaram o Transtorno da Compulsão Alimentar. Para isso, foram analisados 33 estudos triados a partir dos critérios de inclusão e exclusão descritos. Considera-se que os resultados permitiram responder adequadamente aos objetivos propostos nesse estudo, permitindo uma descrição detalhada de aspectos importantes das pesquisas analisadas.

Quanto às características metodológicas apresentadas nos artigos, observa-se que o aumento das produções científicas acerca do TCA ocorreu concomitantemente ao estabelecimento do transtorno enquanto categoria diagnóstica do DSM, em 2013. A análise dos delineamentos dos estudos permitiu identificar que as pesquisas que buscavam a descrição do TCA enquanto categoria diagnóstica, também promoveram a investigação sobre a eficácia de tratamentos diversos para o quadro, na medida em que observa-se uma maior frequência no uso de ensaios clínicos randomizados próximo à publicação do DSM-V. (APA, 2013).

A amostra utilizada nos estudos incluídos vai ao encontro com a literatura que indica que, apesar da ocorrência ser mais comum em mulheres, essa não é expressivamente superior à ocorrência em indivíduos do sexo masculino. Quando comparado aos índices da Bulimia Nervosa, a taxa de TCA em mulheres e homens norte-americanos é bem menos assimétrica, podendo ocorrer em $1,6 \%$ e $0,8 \%$ da população de cada sexo, respectivamente (APA, 2013). Além da prevalência, o TCA se difere dos demais transtornos alimentares por ser mais comum em adultos do que em adolescentes, o que parece refletir nas pesquisas incluídas nesse estudo em que apenas uma investigou amostra de adolescentes (Safer, Couturier, \& Lock, 2007).

Quanto à modalidade de intervenção mais utilizada nos estudos incluídos, tem-se que intervenções individuais foram a opção mais frequente das pesquisas analisadas, em relação aos protocolos de intervenção grupais. A diferença entre a efetividade dessas estratégias não foi significativa dentre os estudos utilizados para análise, indicando, possivelmente, que ambas as modalidades podem ser benéficas ao tratamento do transtorno.

Esse estudo permitiu a identificação das estratégias terapêuticas utilizadas para a intervenção com pessoas diagnosticadas com TCA e os resultados encontrados acerca de sua aplicação. Das estratégias terapêuticas, percebe-se um movimento de mudança quanto à 
abordagem utilizada para embasar as intervenções. A Terapia Cognitivo-Comportamental segue sendo a primeira opção de tratamento para casos de TCA, aparecendo como abordagem em 57,5\% dos estudos e através de técnicas em $63,6 \%$. No entanto, terapias comportamentais, em particular a Terapia Comportamental Dialética, apareceram comouma abordagem que poderia diminuir o número de ECA e aumentar a taxa de remissão entre os pacientes diagnosticados com o transtorno referência.

Apesar de resultados significativos terem sido encontrados em apenas seis dos 15 artigos - cujo objetivo foi verificar a eficácia de estratégias terapêuticas os dados indicaram que a TCC mostra evidência de eficácia no tratamento não somente dos principais sintomas do TCA, como também de comorbidades. A Terapia Cognitivo-comportamentalbaseia-se no pressuposto de que crenças disfuncionais estão associadas ao comer compulsivo e objetiva a modificação e a reestruturação dessas crenças, além disso, emprega técnicas comportamentais para alterar padrões comportamentais relacionados ao transtorno (Duchesne et al., 2007).

Com relação aos instrumentos de avaliação psicológica utilizados nas pesquisas analisadas, é possível destacar (em decorrência de sua maior frequência) quatro instrumentos: o Eating Disorder Examination (EDE), o Beck Depression Inventory (BDI), a Structured Clinical Interview for the DSM (SCID) e a Rosenberg Self-Esteem Scale (RSE). Destaca-se a frequência no uso de instrumentos para avaliar as comorbidades do transtorno (BDI, SCID e RSE), apontando, possivelmente, para a gravidade das características incapacitantes do transtorno. Os estudos incluídos nesta revisão reiteram, portanto, as comorbidades apresentadas no DSM-V (APA, 2013).

\section{Considerações finais}

Essa revisão sistemática da literatura objetivou descrever os instrumentos de avaliação de TCA e as técnicas empregadas nas intervenções psicológicas realizadas com portadores de Transtorno da Compulsão Alimentar. Os resultados sugerem um interesse crescente e global pelo tema da Compulsão Alimentar, tanto no que diz respeito ao diagnóstico quanto às intervenções. A maioria dos estudos revisados são americanos e com delineamento de ensaio clinico randomizado.

A terapia cognitivo-comportamental e as técnicas derivadas dessa abordagem foram as estratégias de intervenção mais citadas e, alguns estudos, identificaram evidências de eficácia nos grupos experimentais. No entanto, observou-se o interesse em investigar intervenções baseadas em outras perspectivas teóricas, como Terapia Comportamental Dialética, sugerindo uma necessidade em desenvolver estratégias alternativas para o TCA e sua sintomatologia. É possível que a alteração diagnóstica ocorrida no DSM 5 (APA, 2013) impulsione o desenvolvimento dessas estratégias,consolidando protocolos estruturados de intervenção.

Algumas limitações foram encontradas ao longo da realização do estudo, como, por exemplo, o recorte idiomático realizado devido às capacidades de compreensão e leitura dos autores do artigo. Diversos idiomas não foram incluídos devido à restrição de leitura em inglês e espanhol. Além disso, apesar de serem selecionadas as principais bases de dados, pode ser entendido como uma limitação do estudo a não inclusão de outras bases na presente pesquisa. Isso pode balizar os resultados encontrados no estudo.

Com relação à publicação nacional, observou-se que nenhum artigo científico brasileiro atendeu aos critérios para a inclusão nesse estudo. Esse dado indica a necessidade de mais pesquisas sobre o tema no Brasil que objetivem investigar a eficácia de intervenções consideradas referencia em outras culturas, como a TCC e o desenvolvimento de instrumentos de avaliação psicológica e diagnóstica validados para a população brasileira. A realização de estudos utilizando delineamentos como ensaio clínico randomizado ou estudos de caso sistemáticos contribuiriam de forma significativa para a produção de conhecimento na área. Diante desse cenário brasileiro, considera-se que essa revisão sistemática da literatura poderá contribuir para o planejamento de pesquisas sobre TCA na medida em que oferece uma descrição detalhada dos delineamentos, instrumentos, intervenções e resultados das investigações realizadas sobre o tema em outros países.

\footnotetext{
Referências

American Psychatric Association. (1994). Diagnostic and Statistcal Manual Of Mental Disorders, (DSM-IV), Washington, DC, American Psychatric Association, 1994.

American Psychiatric Association. (2002). Manual diagnóstico e estatístico de transtornos mentais: texto revisado (DSM-IV-TR). Artmed.
} 
American Psychiatric Association. (2014). Manual diagnóstico e estatístico de transtornos mentais (DSM-5). Porto Alegre: Artmed.

Boggiano, M. M., Wenger, L. E., Burgess, E. E., Tatum, M. M., Sylvester, M. D., Morgan, P. R., \& Morse, K. E. (2017). Eating tasty foods to cope, enhance reward, socialize or conform: What other psychological characteristics describe each of these motives? Journal of health psychology, 22(3), 280-289. https://doi.org/10.1177/1359105315 600240

Chen, E. Y., Cacioppo, J., Fettich, K., Gallop, R., McCloskey, M. S., Olino, T., \& Zeffiro, T. A. (2017). An adaptive randomized trial of dialectical behavior therapy and cognitive behavior therapy for binge-eating. Psychological medicine, 47(4), 703-717. https://doi.org/10.1017/S0033291716002543

Ciarrochi, J., Bilich, L., \& Godsel, C. (2010). Psychological flexibility as a mechanism of change in Acceptance and Commitment Therapy. In Ruth Baer's (Ed). Assessing Mindfulness and Acceptance: Illuminating the Processes of Change (pp. 51-76). New Harbinger Publications, Inc.: Oakland, CA.

Cooper, Z. \& Fairburn, C. (1987). The eating disorder examination: A semi-structured interview for the assessment of the specific psychopathology of eating disorders. International Journal of Eating Disorders, 6(1), 1-8. https://doi. org/10.1002/1098-108X(198701)6:1<1::AID-EAT2260060102>3.0.CO;2-9

Duchesne, M., Appolinário, J. C., Rangé, B. P., Freitas, S., Papelbaum, M., \& Coutinho, W. (2007). Evidências sobre a terapia cognitivo-comportamental no tratamento de obesos com transtorno da compulsão alimentar periódica. Revista de Psiquiatria do Rio Grande do Sul, 29(1), 80-92. https://doi.org/10.1590/S0101-81082007000100015

Hudson JI, Hiripi E, Pope HG Jr, \& Kessler R. C. (2007). The prevalence and correlates of eating disorders in the National Comorbidity Survey Replication. Biological psychiatry, 61(3), 348-358. https://doi.org/10.1016/j. biopsych.2006.03.040

Jacobi F, Wittchen HU, Holting C, et al. (2004). Prevalence, co-morbidity and correlates of mental disorders in the general population: results from the German Health Interview and Examination Survey (GHS). Psychological Medicine, 34(4), 597-611. https://doi.org/10.1017/S0033291703001399

Jansen, A. (2016). Eating disorders need more experimental psychopathology, Behaviour research and therapy, 86, 2-10. https://doi.org/10.1016/j.brat.2016.08.004

Klump, K. L., Bulik, C. M., Kaye, W. H., Treasure, J., \& Tyson, E. (2009). Academy for eating disorders position paper: Eating disorders are serious mental illnesses. International Journal of Eating Disorders, 42, 97-103. https://doi. org/10.1002/eat.20589

Neumark-Sztainer D. \& Hannan P. J. (2000). Weight-related behaviors among adolescent girls and boys: results from a national survey. Archives of Pediatrics \& Adolescent Medicine, 154(6), 569-577. https://doi.org/10.1001/ archpedi.154.6.569

Nunes, R. M. (2013). Transtorno da Compulsão Alimentar Periódica (TCAP) e a abordagem da Terapia Cognitiva Comportamental (TCC). Retrieved from: http://www.ufjf.br/renato_nunes/files/2013/01/Artigo-final-TranstornosAlimentares-0803.pdf.

Rosenberg, M. (1965). Rosenberg self-esteem scale (RSE). In Acceptance and commitment therapy: Measures package $(61,52)$. Retrieved from http://www.integrativehealthpartners.org/downloads/ACTmeasures.pdf\#page=61.

Safer, D. L., Couturier, J. L., \& Lock, J. (2007). Dialectical behavior therapy modified for adolescent binge eating disorder: A case report. Cognitive and behavioral practice, 14(2), 157-167. https://doi.org/10.1016/j.cbpra.2006.06.001

Schulte, E. M., Grilo, C. M., \& Gearhardt, A. N. (2016). Shared and unique mechanisms underlying binge eating disorder and addictive disorders. Clinical psychology review, 44, 125-139. https://doi.org/10.1016/j.cpr.2016.02.001

Spitzer, R. L., Williams, J. B. W., Gibbon, M., \& First, M. B. (1992). The Structured Clinical Interview for DSM-III-R (SCID). I: History, rationale, and description. Archives of General Psychiatry, 49, 624-629. https://doi.org/10.1001/ archpsyc.1992.01820080038006

Striegel-Moore, R. H. \& Franko, D. L. (2008). Should binge eating disorder be included in the DSM-V? A critical review of the state of the evidence. Annu. Rev. Clin. Psychol., 4, 305-324. https://doi.org/10.1146/annurev. clinpsy.4.022007.141149

Stunkard, A. J. (1959). Eating patterns and obesity. Psychiatric Quarterly, 33(2), 284-295. https://doi.org/10.1007/ BF01575455

Tafarodi, R. W. \& Milne, A. B. (2002). Decomposing global self-esteem. Journal of Personality, 70, 443-483. https:// doi.org/10.1111/1467-6494.05017

Treasure, J., Claudino, A. M., \& Zucker, N. (2010). Eating disorders. The Lancet, 375, 583-593. https://doi.org/10.1016/ S0140-6736(09)61748-7

Wang, Y-P., Argimon, I. I. L., \& Werlang, B. S. G. (2011). BDI-II: Inventário de Depressão de Beck II [Manual]. São Paulo: Casa do Psicólogo.

Wilfley, D. E., Bishop, M. E., Wilson, G. T., \& Agras, W. S. (2007). Classification of eating disorders: Toward DSM-V. International Journal of Eating Disorders, 40(S3). https://doi.org/10.1002/eat.20436

Williams, J. B. W., Gibbon, M., First, M. B., Spitzer, R. L., Davies, M., Borus, J., Howes, M. J., Kane, J., Pope, H. G., \& Rounsaville, B. (1992). The Structured Clinical Interview for DSM-III-R (SCID) II. Multi-site test-retest reliability. Archives of General Psychiatry, 49, 630-636. https://doi.org/10.1001/archpsyc.1992.01820080038006 
Wright, R. W., Brand, R. A., Dunn, W., \& Spindler, K. P. (2007) How to Write a Systematic Review. Clinical Orthopaedics And Related Research, 455, 23-29 https://doi.org/10.1097/BLO.0b013e31802c9098

Yager, J. \& Powers, P. S. (2009). Manual Clínico dos Transtornos da Alimentação. Artmed.

Dados dos autores:

Giovanna Nunes Cauduro - Mestranda, Universidade Federal do Rio Grande do Sul.

Gabriel Machado Paz - Graduando, Universidade Federal de Ciências da Saúde de Porto Alegre.

Janaína Thaís Barbosa Pacheco - Pós-Doutorado, Universidade Federal de Ciências da Saúde de Porto Alegre.

Endereco para correspondência:

Janaína Thaís Barbosa Pacheco

Rua Sarmento Leite, 245, sala 207 - Centro

90050-170 Porto Alegre, RS, Brasil

$<$ janainapacheco@uol.com.br>

Recebido em: 28.08.2017

Aceito em: 20.05.2018 